\title{
Korean Translation of the GRADE Series Published in the BMJ, 'GRADE: Incorporating Considerations of Resources Use into Grading Recommendations' (A Secondary Publication)
}

\author{
Translated by: Hong Wook Kim ${ }^{1}$, Jae Hung Jung ${ }^{2,3}$, Do Kyung Kim ${ }^{4}$, Ho Won Kang ${ }^{5}$, Ja Yoon Kü ${ }^{6}$, Hyun Jin Jung ${ }^{7}$, \\ Eu Chang Hwang ; ${ }^{8}$ Guideline Development Committee in the Korean Association of Urogenital Tract Infection and Inflammation \\ ${ }^{1}$ Department of Urology, College of Medicine, Konyang University, Daejeon, ${ }^{2}$ Department of Urology, ${ }^{3}$ Institute of Evidence Based Medicine, \\ Yonsei University Wonju College of Medicine, Wonju, ${ }^{4}$ Department of Urology, Soonchunhyang University Seoul Hospital, Soonchunhyang \\ University College of Medicine, Seoul, ${ }^{5}$ Department of Urology, Chungbuk National University College of Medicine, Cheongju, ${ }^{6}$ Department of \\ Urology, Pusan National University Hospital, Busan, ${ }^{7}$ Department of Urology, Daegu Catholic University School of Medicine, Daegu, \\ ${ }^{8}$ Department of Urology, Chonnam National University Hwasun Hospital, Chonnam National University Medical School, Hwasun, Korea
}

This article is the fifth translation of a GRADE series published in the BMJ for incorporating the considerations of resources use into grading recommendations. Clinical recommendations inevitably involve judgments about the allocation of resources use (costs). Although costs differ from typical healthcare outcomes, such as mortality, morbidity, and quality of life, costs are another potentially important outcome that differs across and within a jurisdiction. A balance sheet is a useful method for determining if the net benefits are worth the incremental costs. Resource use, not just monetary values, should always be presented in an evidence profile. Formal economic modeling may or may not help judge the certainty of the evidence for resource use.

Copyright $\subset$ 2020, Korean Association of Urogenital Tract Infection and Inflammation. All rights reserved. (C) (1) (\$) This is an open access article distributed under the terms of the Creative Commons Attribution Non-Commercial License (http://creativecommons.org/licenses/by-nc/4.0) which permits unrestricted non-commercial use, distribution, and reproduction in any medium, provided the original work is properly cited.
Received: 8 August, 2020

Revised: 8 August, 2020

Accepted: 8 August, 2020

Correspondence to: Eu Chang Hwang

(iD) https://orcid.org/0000-0002-2031-124X

Department of Urology, Chonnam National University Hwasun Hospital, Chonnam National University Medical School, 322 Seoyang-ro, Hwasun-eup, Hwasun 58128, Korea

Tel: +82-62-379-7747, Fax: +82-62-379-7745

E-mail: urohwang@gmail.com

This article is the secondary publication (complete translation in Korean) of the article originally published in the $B M J$ in English (Incorporating considerations of resources use into grading recommendations. 2008;336:1170-3). The Editorin-Chief of Urogenital Tract Infection decided to publish this secondary publication for the reader's sake, and it was approved by $B M J$. BMJ Publishing Group takes no responsibility for the accuracy of the translation from the published English language original and is not liable for any errors that may occur.

\section{GRADE: 자원 사용을 고려한 권고의 도출}

진료지침 개발패널은 개별 환자에 대한 결정에 영향을 미치 는 자원 사용에 대해 여러 가지 다른 옵션을 가지고 있습니다. 의료 비용이 증가함에 따라 자원 사용에 대한 고려가 더욱 중요해지지만 패널은 이러한 고려 사항을 다루기가 어려울 수 있습니다.

권고안 작성에 대한 GRADE (Grading of Recommendations Assessment, Development and Evaluation) 접 근 방식을 설명하는 이 시리즈의 마지막 부분에서는 진료지침 개발패널 및 임상 의사가 자원 사용(resource use)과 관련된 문제를 권고 및 실무에 통합할 수 있는 방법을 살펴볼 것입니
다. 임상적 권고는 불가피하게 자원 배정에 대한 판단, 일반적 으로 비용이라고 불리는 판단을 포함합니다. 이 논문에서는 비용 고려 시 고려해야 할 몇 가지 과제를 다루고, 비용만이 아닌 자원 사용에 초점을 둔 이유를 설명하고, 자원 사용 고려 사항을 권고에 통합하는 방법에 대해 논의합니다.

\section{1. 건강 결과(Outcome)로서의 비용을 고려하는 것은 힘 든 과정입니다. \\ 어떤 의미에서 비용은 사망률, 이환율 및 삶의 질처럼 잠재 적으로 중요한 또 다른 건강 결과로서 환자의 문제를 관리하는 대안들(alternative ways)과 관련이 있습니다. 이러한 임상 적 건강 결과에 미치는 영향과 더불어 중재(intervention)는}


비용을 증가시키거나 감소시킬 수 있습니다. 그러나 비용은 다른 건강 결과들과 여러 가지 측면에서 다릅니다(Box) [1]. 본 시리즈는 치료에 대한 권고에서 고려되어야 할 건강 결과로 서 비용의 정당한 생략을 포함하여 비용과 다른 건강 결과와의 차이점이 내포하고 있는 상이점을 설명하고자 합니다.

\section{2. 효과와 비용을 비교하기 위해 결과요약표(Balance Sheet)를 사용해야 합니다.}

이러한 차이에도 불구하고, 자원 사용에 대한 접근 방식은 의사 결정자가 치료군과 대조군 사이의 차이를 추정해야 한다 는 점에서 다른 건강 결과의 접근방식과 유사합니다. 결과요약 표는 자원 사용의 방향성(예 증가)을 포함하여 치료 옵션의 장단점을 나타내는 간단하지만 유용한 방법입니다[2]. Table 1 과 2는 자간전증(pre-eclampsia)이 있는 여성에서 황산 마그네슘의 유용성을 조사하는 (33개국에서 수행된) 대규모 국제 임상 연구와 관련된 경제학적 분석에서 제시된 근거요약

Box. 비용은 다른 건강 결 과들(other health outcomes)과 어떻게 다른 가?

- 환자는 의료의 긍정적 효과(health benefit)와 부정적 효과(adverse health outcome)를 경험하지만, 의료 비용은 고용주, 환자, 일 반적 으로 (정부로 대표되는) 사회 전체가 부담합니다.

- 비용이 환자의 치료에 대한 임상 의사의 결정에 영향을 미칠지 여부 에 대한 견해(attitude)가 다릅니다.

- 의료 비용은 심지어 동일 행정구역 내에서도 매우 다양하며 시간이 지남에 따라 빠르게 변할 수 있습니다.

- 국가마다 의료 자원의 사용에 대한 기회비용은 크게 다릅니다. 고가 약의 연 처방 비용은 미국에서 한 명의 간호사 급여에 해당할 수 있 지만 중국에서는 30 명의 간호사 급여에 해당할 수 있습니다.

- 보건의료 지출이 다른 부분의 지출을 요구할 때, 보건의료체계, 공 공 지출 또는 사회 전체가 얼마나 부담을 져야 하는지에 따라 다른 견해를 보입니다.

- 자원 사용과 관련된 문제는 매우 정치적이며 진료지침 개발패널에 대한 이해 상충이 발생할 수 있습니다(예, 패널들은 산업 또는 정부 와 관련이 있을 수 있습니다). 표(evidence profile)의 예를 보여줍니다(Tables 1, 2 and Appendices 1, 2) [3-5].

\section{3. 근거요약표는 금전적 가치가 아니라 자원 사용을 제시해 야 합니다.}

지침 개발자는 최적의 비용 추정치(best estimate of costs)가 아니라 자원 사용의 최상의 추정치를 상세히 기록하 는 것이 좋습니다. 비용은 소비된 자원과 자원 단위당 비용의 함수(cost per unit of resource)입니다. 주어진 단위 비용의 변이도(variability)가 넓다는 것을 고려할 때, 단지 총 비용만 보고하게 되면 보건의료인이 처한 각각의 의료 환경에 적용하 는 단위 비용 추정치를 판단하는 데 필요한 정보를 얻지 못하게 됩니다.

또한, 대체 치료(alternative management strategies)에 의해 소비되는 자원을 구체화함으로써 보건의료인은 자원 사용이 자신의 의료 환경에서 실제 패턴을 반영하는지 여부를 판단하고 가장 관련성이 높은 항목에 집중할 수 있게 합니다 (예, 약국에서 약제비 또는 병원 관리자에게 병원비). 마지막으 로, 만일 비용이 최종적으로 사용될 자원에 할당된다면 보건의 료인은 각각의 의료 환경에 필요한 단위 비용을 확인할 수 있고 그렇지 않다면 대체 단위 비용을 확인할 수 있습니다.

Table 1 과 2 는 자원 사용과 각각의 의료 환경을 구체화하는 것의 중요성을 보여줍니다. 황산 마그네슘과 관련된 투약 및 병원비의 차이는 총 국민 소득에 따라 변동이 큽니다. 근거요약 표는 차이점들을 상세히 기록했지만 많은 경제적 분석들은 그렇지 못했습니다. 만일 자원 사용이 구체화되지 않는다면, 보건의료인은 각각의 의료 환경에서 중재와 관련된 비용 증가 를 추정할 수 없습니다(Tables 1, 2 and Appendices 1, 2) [5].

Table 1. Summary of findings on whether clinicians should use magnesium sulfate to prevent eclampsia: clinical outcomes

\begin{tabular}{|c|c|c|c|c|c|c|}
\hline Outcome & $\begin{array}{c}\text { Severity of } \\
\text { pre-eclampsia }\end{array}$ & $\begin{array}{l}\text { Typical control } \\
\text { group risk }\end{array}$ & Typical absolute effect $(95 \% \mathrm{Cl})$ & Relative risk $(95 \% \mathrm{Cl})$ & $\begin{array}{c}\text { No. of } \\
\text { participants }\end{array}$ & $\begin{array}{l}\text { Quality of } \\
\text { evidence }\end{array}$ \\
\hline \multirow[t]{2}{*}{ Eclampsia } & Severe $^{\text {a) }}$ & $27 / 1,000$ & 16 fewer/1,000 (11 to 19$)$ & $0.41(0.29$ to 0.58$)$ & 11,444 & High $^{\text {b) }}$ \\
\hline & Not severe & $15 / 1,000$ & 9 fewer/1,000 (6 to 11$)$ & & & \\
\hline \multirow[t]{2}{*}{ Maternal death } & Severe & $6 / 1,000$ & 3 fewer $/ 1,000$ (0.6 more to 4 fewer $)$ & $0.54(0.26$ to 1.10$)$ & 10,795 & Moderate $^{\mathrm{c})}$ \\
\hline & Not severe & $3 / 1,000$ & 1 fewer $/ 1,000$ ( 0.3 more to 2 fewer $)$ & & & \\
\hline Side effects ${ }^{\mathrm{d})}$ & Severe and not severe & $46 / 1,000$ & 196 more/1,000 (165 to 231$)$ & 5.26 (4.59 to 6.03$)$ & 9,992 & High $^{\text {b) }}$ \\
\hline
\end{tabular}

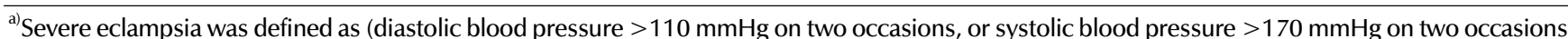
and proteinuria $>3+$ ) or (diastolic blood pressure $>100 \mathrm{mmHg}$ on two occasions, or systolic blood pressure $>150 \mathrm{mmHg}$ on two occasions and proteinuria $>2+$ and at least two signs or symptoms of imminent eclampsia) or for women who had an antihypertensive in the 48 hours before randomization: (in 48 hours before trial entry, highest diastolic blood pressure $>110 \mathrm{mmHg}$, or highest systolic blood pressure $>170 \mathrm{mmHg}$ and proteinuria $>3+$ at trial entry) or (in 48 hours before trial entry, highest diastolic blood pressure $>100 \mathrm{~mm} \mathrm{Hg}$, or highest systolic blood pressure $>150$

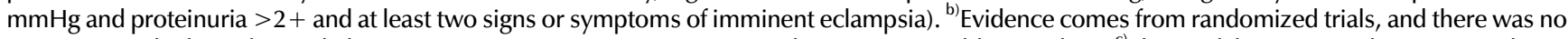
reason to grade down for study limitations, imprecision, inconsistency, indirectness, or publication bias. ${ }^{\mathrm{c}}$ The confidence interval $(\mathrm{Cl})$ was wide, so

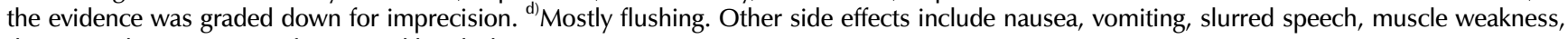
dizziness, drowsiness, confusion, and headache.

Adapted from the article of Guyatt et al. BMJ 2008;336:1170-3 [5].

See Appendix 1 (complete translate in Korean). 
Table 2. Summary of findings on whether clinicians should use magnesium sulfate to prevent eclampsia: resource use viewed from the perspective of the health system

\begin{tabular}{|c|c|c|c|c|c|}
\hline Resource & $\operatorname{Cost}^{\mathrm{a})}$ & $\begin{array}{l}\text { Typical absolute effect } \\
\qquad(95 \% \mathrm{Cl})\end{array}$ & $\begin{array}{l}\text { No. of } \\
\text { participants } \\
\text { (studies) }\end{array}$ & $\begin{array}{l}\text { Quality of } \\
\text { evidence }\end{array}$ & Comment \\
\hline \multicolumn{6}{|c|}{ Magnesium sulfate ampoules $(6 \times 10 \mathrm{ml}$ ampoules/patient) } \\
\hline \multicolumn{6}{|l|}{ Setting: } \\
\hline High-income countries & \multicolumn{2}{|l|}{$\$ 20$ more/patient } & 9,996 & High $^{\text {b) }}$ & \\
\hline Middle-income countries & \multicolumn{2}{|l|}{$\$ 3$ more/patient } & & & \\
\hline Low-income countries & \multicolumn{2}{|c|}{$\$ 5$ more/patient } & & & \\
\hline \multicolumn{6}{|c|}{ Administration of magnesium sulfate ( 1 ampoule/patient) } \\
\hline \multicolumn{6}{|c|}{ Setting: } \\
\hline High-income countries & \multicolumn{2}{|l|}{$\$ 66 /$ patient } & 9,996 & High $^{\text {b) }}$ & $\begin{array}{l}\text { Resources for giving magnesium sulfate } \\
\text { included midwives' time (main cost), } \\
\text { intravenous cannula or needles, sy- } \\
\text { ringes, intravenous fluids, and the drug }\end{array}$ \\
\hline Middle-income countries & \multicolumn{2}{|l|}{ \$14/patient } & & & \\
\hline Low-income countries & \multicolumn{2}{|l|}{$\$ 8 /$ patient } & & & \\
\hline \multicolumn{6}{|c|}{ Other hospital resources (varied widely) } \\
\hline \multicolumn{6}{|c|}{ Setting: } \\
\hline High-income countries & $\$ 12,839$ & $\$ 20$ less/patient ( $\$ 0$ to $\$ 60)$ & 9,996 & Moderate $^{c)}$ & $\begin{array}{l}\text { Use of other hospital resources varied } \\
\text { greatly in both intervention and control } \\
\text { groups. Other hospital costs have been } \\
\text { adjusted for based on the influence of } \\
\text { eclampsia to control for the many other } \\
\text { factors that influenced these costs }\end{array}$ \\
\hline Middle-income countries & $\$ 1,416$ & $\$ 4$ less/patient ( $\$ 0$ to $\$ 10)$ & & & \\
\hline Low-income countries & $\$ 157$ & $\$ 2$ less/patient ( $\$ 1$ to $\$ 3$ ) & & & \\
\hline
\end{tabular}

a) $\$ 1=£ 0.5=€ 0.7$. ${ }^{\mathrm{b})}$ Evidence comes from randomized trials, and there was no reason to grade down for study limitations, imprecision, inconsistency, indirectness, or publication bias. ${ }^{\mathrm{C}}$ The confidence interval $(\mathrm{Cl})$ was wide, so the evidence was graded down for imprecision.

Adapted from the article of Guyatt et al. BMJ 2008;336:1170-3 [5].

See Appendix 2 (complete translate in Korean).

\section{4. 구체적인 의료 현실 또는 현황(Specific Context)이 자 원 사용을 고려하는 데 결정적입니다.}

시간의 변화와 보건의료 행정체계에 따른 비용의 극단적인 다양성은 몇 가지 의미를 가집니다. 첫째로, 진료지침 개발패 널은 환자군, 관심 중재의 성격, 비교 중재 및 보건의료체계에 있어 가능한 한 구체적이어야 합니다. 비교 중재의 선택은 경제적 분석에서 특히 문제가 될 수 있습니다. 만일 비교 중재 의 선택이 부적절하다면(예를 들어, 덜 효과적인 치료보다는 치료하지 않는 것) 결론을 호도할 수 있습니다[6].

둘째로, 진료지침 개발패널은 자원 사용 고려를 적당히 무시 할 수 있고 대체 요법의 다른 장단점에 기초하여 권고안을 만들 수 있습니다. 셋째로, 만일 패널이 자원 사용에 대하여 고려한다면, 비용을 반영하기 전에 다른 건강 결과에 관한 근거수준(quality of evidence)을 결정하고 치료의 원하는/ 원하지 않는 효과(advantage and disadvantage)를 비교 평가해야 합니다.

\section{5. 폭넓은 관점이 바람직합니다.}

개념적으로, 단일 병원의 조제실, 개별 병원 또는 건강 관리 기관(health maintenance organisation) 같은 매우 좁은
대상에 대해 권고를 만들 수 있습니다. 혹은, 지역(health region), 국가 또는 국제 단위의 대상으로 권고를 만들 수도 있습니다.

그러나 전반적인 보건의료체계에 대한 지침보다 좁은 관점 에 만족하는 임상진료지침 사용자는 거의 없습니다. 예를 들 어, 공적 자금으로 운영되는 의료 체계에서 발생된 대부분의 비용을 환자 관점에서는 대부분 무시합니다. 약국의 관점에서 는 약물에 의해 예방된 부작용(뇌졸중 또는 심근경색 같은) 결과로 뒤따라오는 비용절감을 무시하고, 병원의 관점에서는 발생 또는 예방된 외래환자 비용을 간과합니다[7].

더 넓은 관점(사회의 관점)은 누가 부담하는지에 관계없이 모든 비용을 포함하기 때문에 가장 포괄적인 관점입니다. 특히 중재가 광범위한 효과(예, 환자의 건강을 향상시키고 가족 간병인의 시간과 비용을 줄여주는 심부전에 대한 중재)를 갖는 다면 이런 관점이 보통 선호됩니다. 비용 효과 분석이 건강 영향(health effects)의 결과(예, 소득의 변화)를 포함해야 하는지는 더 논란의 여지가 있습니다. 경제 지침(economic guidelines)은 전형적인 비용 효과 분석의 일부로서가 아니라 이러한 건강영향의 결과를 개별적으로 제시할 것을 권유합니 다. 
Table 3. Incremental cost for each episode of eclampsia prevented by magnesium sulfate

\begin{tabular}{llc}
\hline \multirow{2}{*}{ National income } & \multicolumn{2}{c}{ Severity of eclampsia } \\
\cline { 2 - 3 } & Severe & Non-severe \\
\hline High & $\$ 4,125$ & $\$ 7,333$ \\
Medium & $\$ 813$ & $\$ 1,444$ \\
Low & $\$ 688$ & $\$ 1,222$ \\
\hline
\end{tabular}

Adapted from the article of Guyatt et al. BMJ 2008;336:1170-3 [5].

See Appendix 3 (complete translate in Korean).

비록 특정 건강 보험(health plan)이 후속의 비용을 부담하 지 않을 수 있지만, 의사 결정자들은 대체 치료와 관련된 장기 적 자원 사용 증가를 알고 있어야 합니다. 마찬가지로, 환자를 돌보는 임상 의사의 책임은 환자와 환자의 가족에 대한 것이지 만, 이 책무는 자원의 제약과 기회 비용-중재에 사용되는 자원은 다른 용도로 사용될 수 없으며 보건의료체계를 적재적 소에 사용할 수 있는 역량에 영향을 줄 수 있습니다-의 더 넓은 맥락에서 수행됩니다.

\section{6. 자원 사용에 대한 근거 수준 판단}

드물지만 심각한 부작용의 근거와 마찬가지로, 자원 사용의 근거는 의료의 효과(중재의 효과)에 대한 근거 이외의 출처에 서 나올 수 있습니다. 이것은 중재에 대한 임상 시험이 자원 사용을 충분히 보고하지 않기 때문일 수 있고, 이는 임상 시험 상황이 우리가 임상 진료에서 기대하는 임상 환경(자원 사용을 포함하여)을 충분히 반영하지 못해서이거나 관련 자원 사용에 대한 근거가 임상 시험 기간을 넘어 확장될 수 있기 때문입니 다.

임상 시험의 상황에서 보고된 자원 사용의 근거수준 평가 (quality assessment)는 이 시리즈의 두 번째 논문에 기술된 바와 같이 다른 건강 결과에 대한 근거수준 평가와 동일합니다 (본 논문의 Table 1 and Appendix 1) [5]. 임상 시험의 다른 건강 결과와 마찬가지로 근거수준은 각각의 사용된 자원 마다 다를 수 있습니다. 예를 들면, 자간전증에서 황산 마그네 슘을 생각할 때, 우리는 다른 병원 자원(hospital resource)을 사용하는 것보다 약물 자체 그리고 약물의 투여방법과 관련된 자원 사용의 근거수준이 더 높습니다(Table 2 and Appendix 2) [5].

\section{7. 규격화된 경제 모델이 도움이 될 수 있습니다.}

규격화된 경제 모델은 달성된 단위 의료 효과당 비용(cost per unit benefit achieved)으로 나타납니다. 예를 들면, 예방된 뇌졸중당 비용(비용 효과 분석[cost effectiveness analysis]); 삶의 질 보정 추가 생존기간당 비용(cost per quality adjusted life year gained; 비용-효용 분석 [cost-utility analysis]); 또는 화폐단위로 평가된 비용과 의
료 효과(비용-편익 분석[cost-benefit analysis]) 같은 자연 단위당 비용(cost per natural unit)이 있습니다. 이들 요약은 판단에 도움이 될 수 있습니다. 불행하게도, 특히 약물들에 대해 발표된 비용 효과 분석은 연구 비뚤림의 가능성이 높으며 [8] 특정 의료 환경에 따라 다릅니다.

따라서 진료지침 개발그룹들은 자체적으로 규격화된 경제 모델 개발을 생각할 수 있습니다. 그러나 이런 옵션을 고려하는 지침 그룹들은 개발에 필요한 전문 지식과 자원이 필요합니다. 대체 치료에 소비되는 자원 사용의 차이가 클수록 중재의 효용성(net benefits)이 증가하는 비용만큼의 가치가 있는지 에 대한 불확실성이 커지고, 자원 사용에 대한 근거수준이 높을수록 완벽한 경제 모델(full economic model)은 의사 결정에 도움이 될 것입니다.

경제 모델 결정과정은 단위 의료 효과당 비용 계산의 복잡성 과 불확실성을 고려하는 데 필요하지만 투명성을 감소시킵니 다. 게다가, 이러한 모델의 우수성은 그 기반이 되는 데이터의 우수성에 기반합니다. 낮은 근거수준의 의료 효과, 부작용, 자원 사용 추정치는 경제 모델 결정과정의 결과를 매우 불확실 하게 할 것입니다.

비용 효과 또는 비용-효용의 통계 모델의 결과에 대한 신뢰 를 평가하기 위해 범주(criteria)를 사용할 수 있습니다 [9-12]. 그러나, 이러한 모델에는 일반적으로 수많은 가정과 모델에 사용된 추정치들에 대한 다양한 근거수준을 포함합니 다. 이러한 이유로 우리는 근거요약표에 비용 효과 또는 비용 효용 모델을 포함하는 것을 추천하지 않습니다. 그러나 이러한 경제 모델들은 관심 중재를 의료 프로그램에 포함시킬 것인지 고려하는 진료지침 개발 패널, 또는 보건정책 결정자 또는 제3의 지불자(third part payers)의 판단에 정보를 제공할 수 있습니다.

Table 1과 2는 우리에게 고소득, 중간 및 저소득 국가 간에 자간전증이 심한(severe) 환자군과 심하지 않은 (non-severe) 군 간의 예방된 자간증(eclampsia)의 횟수당 증가하는 비용을 보여 줍니다(Table 3 and Appendix 3) [5]. 이 경우와 같이 비용 효과 추정치(cost effective estimates)는 신뢰할 만하더라도 적절한 조치에 대한 명확한 답변을 제공하지는 않습니다. 그러나 대부분의 사람들은 심한 자간전증의 경우에서 예방된 자간증의 횟수당 비용을 고려합 니다. 특히 저소득 국가에서는 심하지 않은 자간전증에 대한 결정은 더욱 어렵습니다. 궁극적으로, 의사 결정자들은 보건 의료체계 또는 사회가 황산 마그네슘 투여에 자원 사용을 할당에 따른 효과와 자간전증 예방의 상대적 가치를 비교 평가해야 합니다. 
끝맺는 말

임상적 의사 결정은 복잡합니다. 진료지침은 다양한 선택지 를 가진 임상 의사와 환자가 치료의 질을 향상시키고 제한된 자원을 최대한으로 사용할 수 있게 도와줍니다. 진료지침이 잘못된 정보보다 제대로 된 정보를 제공하도록 하기 위해 진료지침은 최상의 가능한 근거(best available evidence)에 기반하고 진료지침 개발패널은 근거수준을 평가하고, 근거로 부터 권고를 도출하고 자원 사용을 권고에 통합하는 데 체계적 이고 투명한 과정에 기반하여야 합니다.

임상 의사와 환자는 근거수준과 권고강도를 분명하게 평가 하기 위해 출간된 이 시리즈를 통해 만들어진 진료지침에 의해 최상의 지원을 받을 것입니다. 의료 현장 최일선의 임상 의사 또는 지역적 진료지침을 만드는 사람들은 숙련된 진료지 침 개발자가 수행한 작업을 반복할 필요가 없습니다. 그러나 진료지침을 가장 잘 이용하기 위해서 그들은 진료지침의 기초 가 되는 근거와 판단을 이해해야 합니다. 기본 근거수준과 권고강도에 대한 평가를 포함한 간결한 요약을 이용할 수 있어야 하며 근거수준과 권고강도의 의미와 임상 의사 결정과 정에 미치는 영향을 이해해야 합니다.

\section{CONFLICT OF INTEREST}

No potential conflict of interest relevant to this article are reported.

\section{AUTHOR CONTRIBUTIONS}

H.W.K.: translating the article, and drafting the manuscript, D.K.K., J.Y.K., H.J.J., E.C.H., and H.W.K.: helping to translate and draft the manuscript, J.H.J.: contacting BMJ editorial office to get the approval, helping to translate and draft the manuscript, and final approval.

\section{ORCID}

Hong Wook Kim, https://orcid.org/0000-0002-3847-1401 Jae Hung Jung, https://orcid.org/0000-0002-4990-7098 Do Kyung Kim, https://orcid.org/0000-0002-3696-8756 Ho Won Kang, https://orcid.org/0000-0002-8164-4427 Ja Yoon Ku, https://orcid.org/0000-0003-3460-9386 Hyun Jin Jung, https://orcid.org/0000-0002-1895-7180 Eu Chang Hwang, https://orcid.org/0000-0002-2031-124X

\section{REFERENCES}

1. Guyatt G, Baumann M, Pauker S, Halperin J, Maurer J, Owens $\mathrm{DK}$, et al. Addressing resource allocation issues in recommendations from clinical practice guideline panels: suggestions from an American College of Chest Physicians task force. Chest 2006;129:182-7.

2. Eddy DM. Comparing benefits and harms: the balance sheet. JAMA 1990;263:2493, 498, 501 passim.

3. Altman D, Carroli G, Duley L, Farrell B, Moodley J, Neilson J, et al.; Magpie Trial Collaboration Group. Do women with pre-eclampsia, and their babies, benefit from magnesium sulphate? The Magpie trial: a randomised placebo-controlled trial. Lancet 2002;359:1877-90.

4. Simon J, Gray A, Duley L; Magpie Trial Collaborative Group. Cost-effectiveness of prophylactic magnesium sulphate for 9996 women with pre-eclampsia from 33 countries: economic evaluation of the Magpie trial. BJOG 2006;113:144-51.

5. Guyatt GH, Oxman AD, Kunz R, Jaeschke R, Helfand M, Liberati A, et al.; GRADE Working Group. Incorporating considerations of resources use into grading recommendations. BMJ 2008;336:1170-3.

6. Montori VM, Jaeschke R, Schünemann HJ, Bhandari M, Brozek JL, Devereaux PJ, et al. Users' guide to detecting misleading claims in clinical research reports. BMJ 2004;329:1093-6.

7. Luce BR, Manning GE, Siegel JE, Lipscomb J. Estimating costs in cost-effectiveness analysis. In: Gold MR, Siegel JE, Russell LB, Weinstein MC, editors. Cost-effectiveness in health and medicine. New York: Oxford University Press; 1996. p. 176-213.

8. Friedberg M, Saffran B, Stinson TJ, Nelson W, Bennett CL. Evaluation of conflict of interest in economic analyses of new drugs used in oncology. JAMA 1999;282:1453-7.

9. Garber AM, Phelps CE. Economic foundations of cost effective analysis. J Health Econ 1997;16:1-31.

10. Owens DK. Interpretation of cost-effectiveness analyses. J Gen Intern Med 1998;13:716-7.

11. Gold MR, Siegel JE, Russell LB, Weinstein MC. Costeffectiveness in health and medicine. New York: Oxford University Press; 1996.

12. O'Brien B, Drummond M, Richardson WS, Levine M, Heyland D, Guyatt G. Economic analysis. In: Guyatt G, Rennie D, editors. Users' guides to the medical literature: essentials of evidence-based clinical practice. Chicago: AMA Press; 2002. p. 621-44. 
Appendix 1. 임상의가 자간증을 예방하기 위해 황산 마그네슘을 사용해야 하는지에 대한 연구 결과 요약: 임상 결과

\begin{tabular}{|c|c|c|c|c|c|c|}
\hline 결과 & 전자간증의 심각도 & $\begin{array}{l}\text { 전형적 } \\
\text { 대조군 } \\
\text { 위험도 }\end{array}$ & 전형적 절대 효과(95\% Cl) & 상대위험도(95\% Cl) & 참여자 수 & 근거 수준 \\
\hline \multirow[t]{2}{*}{ 자간증 } & 심각 ${ }^{a)}$ & $27 / 1,000$ & 16 이하/1,000 (11 to 19$)$ & $0.41(0.29$ to 0.58$)$ & 11,444 & 높음 ${ }^{b)}$ \\
\hline & 심각하지 않음 & $15 / 1,000$ & 9 이하/1,000 (6 to 11$)$ & & & \\
\hline \multirow[t]{2}{*}{ 모성 사망 } & 심각 & $6 / 1,000$ & 3 이하 1,000 (0.6 이상 4 이하) & $0.54(0.26$ to 1.10$)$ & 10,795 & 중등 ${ }^{c)}$ \\
\hline & 심각하지 않음 & $3 / 1,000$ & 1 이하/1,000 (0.3 이상 2 이하) & & & \\
\hline 부작용d) & 심각과 심각하지 않음 & $46 / 1,000$ & 196 이상/1,000 (165 to 231$)$ & $5.26(4.59$ to 6.03$)$ & 9,992 & 높음b) \\
\hline
\end{tabular}

$\mathrm{Cl}$ : confidence interval.

a) 심한 자간증은 (두 번 측정한 이완기 혈압 $>110 \mathrm{mmHg}$ 거나 두 번의 수축기 혈압 $>170 \mathrm{mmHg}$ 및 단백뇨 >3+) 또는 (두 번 이완기 혈압 > $100 \mathrm{mmHg}$ 또는 수축기 혈압 $>150 \mathrm{mmHg}$ 및 단백뇨 $>2+$ 그리고 임박한 자간증의 증상이나 징 후가 적어도 2개인 경 우)로 정의됨. 무작위 배정 전 48시간 내에 항고혈압제를 먹은 여성의 경우 (임상 시험 시작 48 시간 전, 최고 이완기 혈압 $>110 \mathrm{mmHg}$ 또는 최고 수축기 혈압 >170 $\mathrm{mmHg}$ 및 임상 시험 시작 시 단백뇨 $>3$ 번 이상 (시험 시작 전) 또는 (시험 시작 48 시간 전, 최고 이완기 혈압 $>100 \mathrm{mmHg}$ 또는 최고 수축기 혈압 >150 $\mathrm{mmHg}$ 및 단백뇨 $>2+$ 및 적어도 2 개의 임박한 자간증의 증상 또는 징후)로 정의됨. ${ }^{b}$ 근거는 무작위 시험에서 비롯되었으며 연구비뚤림 위험, 비정밀성, 비일 관성, 비직접 성 또는 출판 비뚤림으로 등급을 낮추지 않음. ${ }^{\mathrm{c}}$ 신뢰 구간이 넓음: 비정밀성 등급을 낮춤. ${ }^{\mathrm{d}}$ 대부분 홍조. 다른 부작용으로는 메스꺼움, 구토, 분명하지 않은 말투, 근육 약화, 현기증, 졸음, 혼란 및 두통이 발생.

Appendix 2. 임상의가 자간증을 예방하기 위해 황산 마그네슘을 사용해야 하는지에 대한 연구 결과 요약: 보건 시스템의 관점에서 볼 때 자원 사용

\begin{tabular}{|c|c|c|c|c|c|}
\hline 자원 & 비용 ${ }^{a)}$ & 전형적 절대 효과 $(95 \% \mathrm{Cl})$ & 참여자 수(연구들) & 근거수준 & 비고 \\
\hline \multicolumn{6}{|c|}{$\begin{array}{l}\text { 황산 마그네슘 앰플(6×10 ml 앰플/환자) } \\
\text { 설정: }\end{array}$} \\
\hline 고소득국가 & $\$ 20$ 이상/환자 & & 9,996 & 높음 ${ }^{\prime}$ & \\
\hline 중위소득국가 & $\$ 3$ 이상/환자 & & & & \\
\hline 하위소득국가 & $\$ 5$ 이상/환자 & & & & \\
\hline \multirow{2}{*}{\multicolumn{6}{|c|}{$\begin{array}{l}\text { 황산 마그네슘 투약(1 앰플/환자) } \\
\text { 설정: }\end{array}$}} \\
\hline & & & & & \\
\hline 고소득국가 & \$66/환자 & & 9,996 & 높음 ${ }^{b)}$ & $\begin{array}{l}\text { 황산 마그네슘을 제공하기 위한 자원에는 조 } \\
\text { 산사의 시간(주요 비용), 정맥 내 캐뉼라 또 } \\
\text { 는 바늘, 주사기, 정맥 수액 및 약물이 포함됩 } \\
\text { 니다. }\end{array}$ \\
\hline 중위소득국가 & \$14/환자 & & & & \\
\hline 하위소득국가 & $\$ 8 /$ 환자 & & & & \\
\hline \multirow{2}{*}{\multicolumn{6}{|c|}{$\begin{array}{l}\text { 다른병 원자원(광범위하게 변화) } \\
\text { 설정: }\end{array}$}} \\
\hline & & & & & \\
\hline 고소득국가 & $\$ 12,839$ & $\$ 20$ 미만/환자 $(\$ 0$ to $\$ 60)$ & 9,996 & 중등 ${ }^{c)}$ & $\begin{array}{l}\text { 다른 병원 자원의 사용은 중재 및 대조군 모두 } \\
\text { 에서 크게 달랐습니다. 다른 병원 비용은 이 } \\
\text { 들 비용에 영향을 받는 많은 다른 요소들을 } \\
\text { 조정하기 위해 자간증에의 영향을 근거로 조 } \\
\text { 정되었습니다. }\end{array}$ \\
\hline 중위소득국가 & $\$ 1,416$ & $\$ 4$ 미만/환자 $(\$ 0$ to $\$ 10)$ & & & \\
\hline 하위소득국가 & $\$ 157$ & \$2 미만/환자 $(\$ 1$ to $\$ 3)$ & & & \\
\hline
\end{tabular}

$\mathrm{Cl}$ : confidence interval.

a) $\$ 1=£ 0.5=€ 0.7 .^{b}$ 근거는 무작위 시험에서 비롯되었으며 연구 비뚤림, 비정밀성, 비일관성, 비직접성 또는 출판 비뚤림으로 등급을 낮추지 않음. c)신뢰 구간이 넓음: 비정밀성 등급을 낮춤.

Appendix 3. 황산 마그네슘으로 예방된 자간증의 각 에피소드에 대한 비용 증가

\begin{tabular}{clc}
\hline \multirow{2}{*}{ 국민 소득 } & \multicolumn{2}{c}{ 자간증의 심각도 } \\
\cline { 2 - 3 } & 심각 & 심각하지 않음 \\
\hline 높음 & $\$ 4,125$ & $\$ 7,333$ \\
중간 & $\$ 813$ & $\$ 1,444$ \\
낮음 & $\$ 688$ & $\$ 1,222$ \\
\hline
\end{tabular}

